\title{
The interleukin-13 paradox in asthma: effective biology, ineffective biologicals
}

\author{
Parameswaran Nair and Paul M. O’Byrne
}

Affiliation: Firestone Institute for Respiratory Health, St Joseph's Healthcare and Dept of Medicine, McMaster University, Hamilton, ON, Canada.

Correspondence: Parameswaran Nair, Firestone Institute for Respiratory Health, St Joseph's Healthcare Hamilton, 50 Charlton Avenue East, Hamilton, ON, L8N 4A6, Canada. E-mail: paramesamcmaster.ca

@ERSpublications

IL-13 is involved in almost all aspects of asthma pathobiology, yet, anti-IL-13 mAb clinical trials, including the prednisone-sparing TROPOS study published in this issue of the ERJ, have been ineffective http://ow.ly/ZMnd30n27VN

Cite this article as: Nair P, O'Byrne PM. The interleukin-13 paradox in asthma: effective biology, ineffective biologicals. Eur Respir J 2019; 53: 1802250 [https://doi.org/10.1183/13993003.02250-2018].

Interleukin-13 (IL-13) is a pleiotropic cytokine produced by type-2 helper T-cells (Th2), mast cells and basophils, and is thought to be involved in many of the features of asthma. IL-13 induces many biological responses relevant to asthma, such as B-cell immunoglobulin E production, generation of eosinophil chemoattractants, maturation of mucus-secreting goblet cells, production of extracellular matrix proteins and myofibroblast differentiation, and enhanced contractility of airway smooth muscle cells in response to cholinergic agonists. These cytokines activate the Janus kinase/signal transducer and activator of transcription (JAK/STAT) signalling cascades, which may contribute to allergic responses. In addition, stimulation of the phosphatidylinositol 3-kinase pathway through recruitment of members of the insulin receptor substrate family may contribute to survival and proliferation [1]. IL-13 also stimulates the secretion of periostin, a matricellular protein, which has a role in fibroblast activation and increasing collagen gel elasticity [2]. By activating epithelial nitric oxide synthase through its effect on STAT-6 [3], its biological activity may also be reflected by raised levels of exhaled nitric oxide (FeNO). IL-13 levels in bronchoalveolar lavage fluid, and gene and protein expression in bronchial mucosal tissues are increased in patients with moderate to severe asthma [4]. Thus, IL-13 is potentially involved in every aspect of the pathobiology of asthma. IL-13 is thought to mediate most of its effect through binding to receptors IL-13R $\alpha 1$ and IL-4R $\alpha$. Therefore, it is logical to assume that blocking signalling through either of these receptors would lead to salutary effects in poorly controlled asthma.

As with many drug development processes for asthma, clinical effects of blocking IL-13 with monoclonal antibodies (mAbs) were initially evaluated in a human allergen-challenge model. Anrukinzumab (IMA-638) is an IgG1 monoclonal antibody specific for the IL-13 epitope that binds IL-4R $\alpha$, whereas IMA-026 is specific for the IL-13 epitope that binds IL-13R $\alpha 1$ and IL-13R $\alpha 2$. The effects were modest and inconsistent [5]. While IMA-638 attenuated early allergen responses, neither had an effect on the late responses, airway hyperresponsiveness or sputum eosinophilia induced by the allergen, and the effects were not sustained at later time points that were examined. In a similar study design, lebrikizumab (an IgG4 neutralising mAb against IL-13 and blocks its interaction with IL-4R $\alpha$ ) also had very modest effects on the late allergen response [6]. After promising results in early phase 2 clinical trials in mild to moderate asthma [7], the drug did not demonstrate consistently significant exacerbation reductions in replicate phase 3 clinical trials of patients with moderate to severe asthma (51\% and 26\%, 
respectively), even in those patients with raised serum periostin [8]. A number of other compounds (e.g. GSK679586, QAX576, AMG317) directed against IL-13 failed to demonstrate clinical efficacy in early phase 2 clinical trials.

Tralokinumab, another IgG4 IL-13 neutralising mAb, was not evaluated in an allergen challenge model. Instead, an early phase 2, dose-ranging study (150, 300, $600 \mathrm{mg}$ s.c. every 2 weeks) evaluated its efficacy over a 13-week period in patients with moderate asthma and did not demonstrate a significant effect on the primary outcome of Asthma Control Questionnaire score (ACQ-6; ACQ mean of six individual item scores), while there was a modest improvement in forced expiratory volume in $1 \mathrm{~s}$ [9]. Subsequently, a larger phase $2 \mathrm{~b}$ clinical trial of $300 \mathrm{mg}$ administered either every 2 weeks or every 4 weeks over a 52 -week period did not demonstrate any reduction in exacerbation frequencies compared to placebo [10]. There was a suggestion that the benefits may be greater in those patients with greater baseline bronchodilator reversibility and those who are not on maintenance oral corticosteroids, and in those who had higher levels of serum periostin and dipeptidyl peptidase-4. Tralokinumab (300 mg s.c. every 2 weeks) went on to be evaluated in two large multicentre phase 3 clinical trial in patients with moderate-severe asthma [11]. In the first of these (STRATOS-1), there was overall no significant decrease in annual exacerbation rate compared to placebo. However, the exacerbation rate was significantly reduced in those patients who had raised FeNO. However, in the second large clinical trial (STRATOS-2) that specifically examined the treatment effect of tralokinumab in those patients with a raised FeNO of greater than $37 \mathrm{ppb}$, the previously observed exacerbation rate reduction compared to placebo was not observed.

With the background of this, less than encouraging, clinical history of the molecule, in this issue of the European Respiratory Journal, Busse et al. [12] examined the effects of tralokinumab in a population of patients with more severe asthma who required maintenance oral glucocorticosteroids for controlling their asthma. In the TROPOS clinical trial, 140 patients were randomised to tralokinumab $300 \mathrm{mg}$ or placebo administered subcutaneously every 2 weeks. The primary end-point was percent change from baseline in average oral glucocorticosteroid dose at week 40 , while maintaining asthma control. At week 40 , although the drug was well tolerated, the percent reduction from baseline in the final daily average oral glucocorticosteroid dose was not significantly different between tralokinumab and placebo (37.62\% versus 29.85\%; $\mathrm{p}=0.271$ ). There were no significant between-treatment differences for any of the secondary end-points that were examined. In contrast, clinical trials that have targeted the IL-5 cytokine $[13,14]$ or its receptor [15] have demonstrated significant prednisone-sparing effects. Targeting the IL-4R with dupilumab (an IgG4 mAb), that blocks both IL-4 and IL-13 signalling, has also demonstrated significant reduction in asthma exacerbations [16] and prednisone-sparing effects [17].

These results present a puzzling paradox of dissociation between biological efficacy of IL-13 and lack of efficacy of IL-13 blockade in clinical trials. There are several possible potential explanations. These may relate to the interventions or to the patient selection and study designs. As with any pharmacological intervention, one consideration is the design and development of the mAbs. This is unlikely, as a number of different molecules that were independently developed by various pharmaceutical companies did not show clinical efficacy. However, since there is no information on airway pharmacokinetics of any of these $\mathrm{mAbs}$, it is plausible that these drugs may not achieve enough concentration in the airways, or may have variable elimination rate of the mAb-target complex in the airway that may in turn regulate the degree of free target inhibition [18]. In this regard, it would be interesting to see if an inhaled anti-IL-13 [19] would demonstrate clinical efficacy. A second possibility is the potential relationship between IL-13 gene polymorphisms that may exist in patient populations and clinically relevant biology. For example, a variant with a single nucleotide polymorphism (SNP) in the IL13 gene at position +2044G>A (rs20541) is expected to result in the non-conservative replacement of a positively charged arginine (R) with a neutral glutamine (Q) at position 144. IL-13Q144 is associated with augmented allergic airway inflammation and bronchial asthma remodelling [20]. There is no clinical information relating the effects of IL-13 blockade to the prevalence of any IL-13 gene SNPs.

Assuming that the mAbs are designed appropriately, targeted against the right target epitopes, and are administered in the correct dosages, one has to consider that IL-13 blockade alone is insufficient to improve asthma outcomes as there are many redundant processes in the intricate immunopathobiology of asthma. Additionally, although eosinophils may not play a central effector role in all patients with asthma, it may be necessary to reduce airway eosinophil numbers for an asthma intervention to be effective. Tralokinumab, over a 12-week treatment period, does not reduce effectively either the luminal or tissue eosinophil numbers, as demonstrated in the MESOS clinical trial [21]. One of the reasons that combined IL-4 and IL-13 blockade (with dupilumab) showed clinical efficacy may be the ability to reduce airway eosinophilia by IL-4 blockade. However, this remains to be proven in clinical trials. Finally, identifying the appropriate patient population in whom IL-13 is likely to play a dominant role may be very important, just as choosing patients with significant blood and sputum eosinophils led to successful targeting of IL-5. 
Since the most pronounced biological effects of IL-13 are on goblet cells and airway smooth muscle [22], it may be important to select patients whose symptoms are largely driven by either or both of these features of asthma. Lastly, the role in asthma of signalling through the IL-13R $\alpha 2$, which is believed to an inhibitory subunit of the type II receptor, by acting as a "decoy" receptor for IL-13, is not known.

In conclusion, although IL-13 plays an important role in the pathobiology of asthma, there may be a number of redundant processes that reduce the clinical benefits of targeting IL-13 alone. Currently, there is no approved anti-IL-13 mAb available for the treatment of asthma. There may, however, be a proportion of patients with poorly controlled asthma who benefit from blocking IL-13 signalling. These may be patients with more severe airway hyperresponsiveness and have significant mucus hypersecretion. Identifying these patients using appropriate biomarkers, such as a methacholine or mannitol hyperresponsiveness, computed tomography mucus quantification [23], and raised FeNO (disproportionate to other Th2 markers such as eosinophil numbers) may hold the key to successful demonstration of the efficacy of IL-13 blockade in asthma.

Conflict of interest: P. Nair reports institutional grant support for two clinical trials (one of which is investigator-initiated), consultancy for two advisory board meetings and honoraria for five non-promotional lectures in symposia funded by AstraZeneca, institutional grant support for one clinical trial from Boehringer Ingelheim, institutional grant support for one investigator-initiated clinical trials and honoraria for three non-promotional lectures in symposia funded by Novartis, institutional grant support for two clinical trials (one of which is investigator-initiated), and fees for consultancy for two scientific advisory board meetings from Sanofi, institutional grant support for an investigator-initiated clinical trial, consultancy for three advisory board meetings and honoraria for five non-promotional lectures in symposia funded by Teva, institutional grant support for two investigator-initiated clinical trials and consultancy fees for participating in a scientific meeting from GSK, and personal fees for consultancy from Theravance and Knopp, outside the submitted work. P.M. O'Byrne reports membership of a joint oversight board for LABA safety study; fees for consultancy from AstraZeneca, GSK, Merck and Boehringer; and institutional grants from AstraZeneca, Genentech and Novartis.

Support statement: P. Nair is supported by the Frederick E. Hargreave Teva Innovation Chair in Airway Diseases.

\section{References}

1 Wills-Karp M. Interleukin-13 in asthma pathogenesis. Immunol Rev 2004; 202: 175-190.

2 Takayama G, Arima K, Kanaji T, et al. Periostin: a novel component of subepithelial fibrosis of bronchial asthma downstream of IL-4 and IL-13 signals. J Allergy Clin Immunol 2006; 118: 98-104.

3 Chibana K, Trudeau JB, Mustovich AT, et al. IL-13 induced increases in nitrite levels are primarily driven by increases in inducible nitric oxide synthase as compared with effects on arginases in human primary bronchial epithelial cells. Clin Exp Allergy 2008; 38: 936-946.

4 Saha SK, Berry MA, Parker D, et al. Increased sputum and bronchial biopsy IL-13 expression in severe asthma. J Allergy Clin Immunol 2008; 121: 685-691.

5 Gauvreau GM, Boulet LP, Cockcroft DW, et al. Effects of interleukin-13 blockade on allergen-induced airway responses in mild atopic asthma. Am J Respir Crit Care Med 2011; 183: 1007-1014.

6 Scheerens H, Arron JR, Zheng Y, et al. The effects of lebrikizumab in patients with mild asthma following whole lung allergen challenge. Clin Exp Allergy 2014; 44: 38-46.

7 Corren J, Lemanske RF, Hanania NA, et al. Lebrikizumab treatment in adults with asthma. N Engl J Med 2011; 365: 1088-1098.

8 Hanania NA, Korenblat P, Chapman KR, et al. Efficacy and safety of lebrikizumab in patients with uncontrolled asthma (LAVOLTA I and LAVOLTA II): replicate, phase 3, randomised, double-blind, placebo-controlled trials. Lancet Respir Med 2016; 4: 781-796.

9 Piper E, Brightling C, Niven R, et al. A phase II placebo-controlled study of tralokinumab in moderate-to-severe asthma. Eur Respir J 2013; 41: 330-338.

10 Brightling CE, Chanez P, Leigh R, et al. Efficacy and safety of tralokinumab in patients with severe uncontrolled asthma: a randomised, double-blind, placebo-controlled, phase 2b trial. Lancet Respir Med 2015; 3: 692-701.

11 Panettieri RA Jr, Sjöbring U, Péterffy A, et al. Tralokinumab for severe, uncontrolled asthma (STRATOS 1 and STRATOS 2): two randomised, double-blind, placebo-controlled, phase 3 clinical trials. Lancet Respir Med 2018; 6: 511-525.

12 Busse WW, Brusselle GG, Korn S, et al. Tralokinumab did not demonstrate oral corticosteroid-sparing effects in severe asthma. Eur Respir J 2019; 53: 1800948.

13 Nair P, Pizzichini MM, Kjarsgaard M, et al. Mepolizumab for prednisone-dependent asthma with sputum eosinophilia. N Engl J Med 2009; 360: 985-993.

14 Bel EH, Wenzel SE, Thompson PJ, et al. Oral glucocorticoid-sparing effect of mepolizumab in eosinophilic asthma. N Engl J Med 2014; 371: 1189-1197.

15 Nair P, Wenzel S, Rabe KF, et al. Oral glucocorticoid-sparing effect of benralizumab in severe asthma. $N$ Engl J Med 2017; 376: 2448-2458.

16 Castro M, Corren J, Pavord ID, et al. Dupilumab efficacy and safety in moderate-to-severe uncontrolled asthma. N Engl J Med 2018; 378: 2486-2496.

17 Rabe KF, Nair P, Brusselle G, et al. Efficacy and safety of dupilumab in glucocorticoid-dependent severe asthma. N Engl J Med 2018; 378: 2475-2485.

18 Tiwari A, Kasaian M, Heatherington AC, et al. A mechanistic PK/PD model for two anti-IL13 antibodies explains the difference in total IL-13 accumulation observed in clinical studies. MAbs 2016; 8: 983-990. 
19 Burgess G, Boyce M, Jones $M$, et al. Randomized study of the safety and pharmacodynamics of inhaled interleukin-13 monoclonal antibody fragment VR942. EBioMedicine 2018; 35: 67-75.

20 Nakamura Y, Sugano A, Ohta M, et al. Docking analysis and the possibility of prediction efficacy for an anti-IL-13 biopharmaceutical treatment with tralokinumab and lebrikizumab for bronchial asthma. PLoS One 2017; 12: e0188407.

21 Russell RJ, Chachi L, FitzGerald JM, et al. Effect of tralokinumab, an interleukin-13 neutralising monoclonal antibody, on eosinophilic airway inflammation in uncontrolled moderate-to-severe asthma (MESOS): a multicentre, double-blind, randomised, placebo-controlled phase 2 trial. Lancet Respir Med 2018; 6: 499-510.

22 Wills-Karp M, Luyimbazi J, Xu X, et al. Interleukin-13: central mediator of allergic asthma. Science 1998; 282: 2258-2261.

23 Dunican EM, Elicker BM, Gierada DS, et al. Mucus plugs in patients with asthma linked to eosinophilia and airflow obstruction. J Clin Invest 2018; 128: 997-1009. 